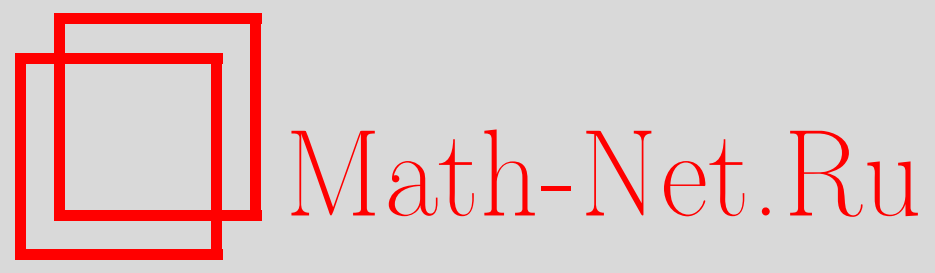

В. В. Назаров, Схемы открытого распределения ключа на основе некоммутативной операции, Дискрет. матем., 2006, том 18, выпуск 4, 148-157

DOI: https://doi.org/10.4213/dm79

Использование Общероссийского математического портала Math-Net.Ru подразумевает, что вы прочитали и согласны с пользовательским соглашением http://www . mathnet.ru/rus/agreement

Параметры загрузки:

IP : 54.209 .52 .79

26 апреля 2023 г., 11:48:34

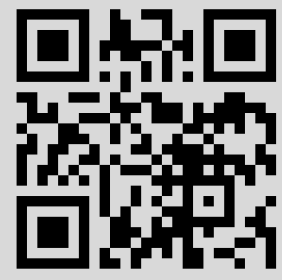




\title{
Схемы открытого распределения ключа на основе некоммутативной операции
}

\author{
() 2006 г. В. В. Назаров
}

\begin{abstract}
В работе изучаются свойства двух схем открытого распределения ключа, основанных на некоммутативной операции и предложенных В. М. Сидельниковым. В качестве некоммутативной операции рассматриваются операция из семейства, предложенного М. А. Черепневым, а именно, операции в кольцах целых чисел круговых полей, основанные на символе степенного вычета. В работе проведен криптоанализ обеих схем для одного частного случая некоммутативной операции. В случае произвольной операции из вышеупомянутого класса доказана нестойкость первой схемы. Для второй схемы доказана эквивалентность задачи ее вскрытия одной задаче вычислительной алгебраической теории чисел.
\end{abstract}

\section{1. Введение}

Список односторонних функций, используемых в криптографии, весьма невелик. Подавляющее большинство криптографических протоколов основано на отсутствии в настоящее время полиномиальных алгоритмов обращения операций умножения целых чисел и возведения в степень в конечных полях. В настоящей работе изучается операция вычисления символа степенного вычета на предмет ее использования в схеме открытого распределения ключа на основе некоммутативной операции.

Принципиальная схема открытого распределения ключа на основе некоммутативной операции была предложена В. М. Сидельниковым [1]. М. А. Черепнев [2] предложил использовать для этой схемы символы Лежандра и Якоби. О. Н. Василенко заметил, что можно использовать и символ степенного вычета (обобщенный символ Якоби). В работах [2, 3] рассмотрены примеры полиномиально вычислимых функций, на основе данного символа, которые оказались намного более стойкими к обращению, чем все предложенное ранее для данной схемы.

\section{2. Используемый математический и криптографический aाIIapat}

Пусть

$$
\mathbf{K}=\mathbf{Q}\left(\zeta_{p}\right)
$$


- круговое поле, где $p$ - простое целое рациональное число,

$$
\zeta_{p}=e^{2 \pi i / p}
$$

Обозначим $\mathbf{R}$ кольцо целых поля $\mathbf{K}$. Известно, что $\mathbf{R}$ является факториальным кольцом и векторным пространством над $\mathbf{Z}$ с базисом, состоящим из степеней $\zeta_{p}$, a именно,

$$
\mathbf{R}=\left\langle 1, \zeta_{p}, \ldots, \zeta_{p}^{p-2}\right\rangle_{\mathbf{z}}
$$

Введем также обозначение

$$
\lambda=1-\zeta_{p}
$$

Известно, что

$$
p=u \cdot \lambda^{p-1}
$$

где $u$ - обратимый элемент кольца $\mathbf{R}$.

Пусть $\mathscr{F}-$ простой идеал в $\mathbf{R}, a \in \mathbf{R}$, причем $\mathscr{F}$ и $(a)$ взаимно просты с $(\lambda)$ и между собой, пусть также $\mathscr{I}$ - идеал в $\mathbf{R}$, взаимно простой с $(a)$ и $(\lambda)$.

Определение 1. Символ степенного вычета (обобщенный символ Якоби) есть

$$
\begin{aligned}
& \left(\frac{a}{\mathscr{g}}\right)_{p}=\zeta_{p}^{i} \equiv a^{(N(\mathscr{g})-1) / p} \quad(\bmod \mathscr{g}), \\
& \left(\frac{a}{\Phi}\right)_{p}=\prod_{\mathscr{g} \mid \Phi, \Phi}\left(\frac{a}{\Phi}\right)_{p}^{\nu_{\mathscr{f}}(\Phi)},
\end{aligned}
$$

где

$$
\mathscr{I}=\prod_{\mathscr{F}} \mathscr{F}^{\nu_{\mathscr{F}}(\Im)}
$$

Свойство 1. Символ степенного вычета мультипликативен по верхнему и по нижнему аргументам (при соблюдении условий взаимной простоты).

Свойство 2. Пусть $a_{1}, a_{2} \in \mathbf{R} \backslash\{0\},\left(a_{1}\right),\left(a_{2}\right)$ взаимно просты с $(p), \Phi$ - идеал, взаимно простой с $\left(a_{i}\right)$ и $(p)$,

$$
a_{1} \equiv a_{2} \quad(\bmod \Phi)
$$

Тогда

$$
\left(\frac{a_{1}}{g}\right)_{p}=\left(\frac{a_{2}}{\mathscr{g}}\right)_{p} .
$$

Определение 2. Пусть $a, b \in \mathbf{R} \backslash\{0\},(a),(b)$ взаимно просты между собой и с $(p)$. Тогда

$$
\left(\frac{a}{b}\right)_{p}=\left(\frac{a}{(b)}\right)_{p} .
$$


Определение 3. Пусть $p$ - простое целое рациональное число, $x \in \mathbf{Z}$ взаимно просто с $p$. Тогда частное Ферма от $x$

$$
\Phi_{p}(x)=\frac{x^{p-1}-1}{p}(\bmod p) .
$$

Легко заметить, что для $x, y \in \mathbf{Z},(x, p)=(y, p)=1$, выполнено соотношение

$$
\Phi_{p}(x y) \equiv \Phi_{p}(x)+\Phi_{p}(y) \quad(\bmod p) .
$$

Далее рассмотрим два варианта схемы открытого распределения ключа на основе некоммутативной операции. Оба варианта были предложены В. М. Сидельниковым в [1].

Пусть $(\mathbf{G}, *)$ - полугруппа, где * - некоммутативная, ассоциативная, полиномиально вычислимая операция, $\mathbf{G}_{0}, \mathbf{G}_{1}, \mathbf{G}_{2}$ - подполугруппы, внутри которых операция * коммутирует, $\mathbf{A}, \mathbf{B}-$ абоненты, $\gamma \notin \mathbf{G}_{0}, \mathscr{K}$ - общий секретный ключ.

\section{Схема I.}

Абонент А

1. выбирает $a_{1}, a_{2} \in \mathbf{G}_{\mathbf{0}}$, вычисляет $d_{A}=a_{1} * \gamma * a_{2}$, отправляет $d_{A}$ абоненту В.

2. вычисляет $\mathscr{K}=\mathscr{K}_{A}=a_{1} * d_{B} * a_{2}$.
Абонент В

1. выбирает $b_{1}, b_{2} \in \mathbf{G}_{\mathbf{0}}$, вычисляет $d_{B}=b_{1} * \gamma * b_{2}$, отправляет $d_{B}$ абоненту $\mathbf{A}$.

2. вычисляет $\mathscr{K}=\mathscr{K}_{B}=b_{1} * d_{A} * b_{2}$.

\section{Схема II.}

\section{Абонент А}

1. выбирает $a_{i} \in \mathbf{G}_{\mathbf{i}}, i=1,2$;

вычисляет $d_{A}=a_{1} * a_{2}$;

отправляет $d_{A}$ абоненту $\mathbf{B}$.

2. Calculate $\mathscr{K}=\mathscr{K}_{A}=a_{1} * d_{B} * a_{2}$.

\section{Абонент В}

1. выбирает $b_{i} \in \mathbf{G}_{\mathbf{i}}, i=1,2$;

вычисляет $d_{B}=b_{1} * b_{2}$;

отправляет $d_{B}$ абоненту $\mathbf{A}$.

2. вычисляет $\mathscr{K}=\mathscr{K}_{B}=b_{1} * d_{A} * b_{2}$.

В [3] показано, что в качестве операции * можно использовать операцию в $\mathbf{K}$

$$
a * b=a \cdot b \cdot\left(\frac{\eta(a)}{\mu(b)}\right)_{p},
$$

где $\mu(x), \eta(x)$ - мультипликативные функции такие, что

$$
\mu\left(\zeta_{p}\right)=\eta\left(\zeta_{p}\right)=1 \text {. }
$$

В частности, при $\eta(x)=N(x)$ (где $N(x)$ - норма из $\mathbf{K}$ в $\mathbf{Q})$ и $\mu(x)=\mathscr{g}^{\Phi_{p}(N(x))}$ получим операцию [2]

$$
a * b=a \cdot b \cdot\left(\frac{N(a)}{\mathscr{J}}\right)_{p}^{\Phi_{p}(N(b))} .
$$

Замечание 1. Вместо $\Phi_{p}(N(x))$ можно взять любую логарифмическую функцию по модулю $p$. 


\section{3. Криптоанализ схем I, II для операции (1)}

Теорема 1. Схема I с операчией (1) нестойкая.

В схеме II с той же операчией * задача нахождения ключа по открытой информачии эквивалентна задаче нахождения по открытой информачии любого из трех элементов

$$
\begin{aligned}
& \tau_{0}=\left(\frac{\eta\left(a_{1}\right)}{\mu\left(d_{B}\right)}\right)_{p} \cdot\left(\frac{\eta\left(d_{B}\right)}{\mu\left(a_{2}\right)}\right)_{p}=\left(\frac{\eta\left(b_{1}\right)}{\mu\left(d_{A}\right)}\right)_{p} \cdot\left(\frac{\eta\left(d_{A}\right)}{\mu\left(b_{2}\right)}\right)_{p}, \\
& \tau_{1}=\left(\frac{\eta\left(a_{2}\right)}{\mu\left(b_{1}\right)}\right)_{p} \cdot\left(\frac{\eta\left(b_{1}\right)}{\mu\left(a_{2}\right)}\right)_{p}^{-1}=\frac{a_{2} * b_{1}}{b_{1} * a_{2}}, \\
& \tau_{2}=\left(\frac{\eta\left(b_{2}\right)}{\mu\left(a_{1}\right)}\right)_{p} \cdot\left(\frac{\eta\left(a_{1}\right)}{\mu\left(b_{2}\right)}\right)_{p}^{-1}=\frac{b_{2} * a_{1}}{a_{1} * b_{2}} .
\end{aligned}
$$

При $\mu=\eta, \tau_{1}=\left(\eta\left(a_{2}\right), \eta\left(b_{1}\right)\right)_{\lambda}, \tau_{2}=\left(\eta\left(b_{2}\right), \eta\left(a_{1}\right)\right)_{\lambda}$ - символы норменного вычета. В этом случае можно считать, что общим секретным ключом, вырабатываемым в схеме, является один заранее выбранный символ норменного вычета, например $\tau_{1}=\left(d_{A} * d_{B}\right) / \mathscr{K}$.

Напомним, что открытой информацией являются элементы $d_{A}$ и $d_{B}$, а также описание коммутирующих подполугрупп $\mathbf{G}_{i}, i=0$ в схеме I и $i=1,2$ в схеме II; кроме того, в схеме I известен элемент $\gamma$.

Доказательство. Сначала докажем первое утверждение теоремы.

Заметим, что

$$
\begin{aligned}
x * y=y * x & \Longleftrightarrow x \cdot y \cdot\left(\frac{\eta(x)}{\mu(y)}\right)_{p}=y \cdot x \cdot\left(\frac{\eta(y)}{\mu(x)}\right)_{p} \\
& \Longleftrightarrow\left(\frac{\eta(x)}{\mu(y)}\right)_{p}=\left(\frac{\eta(y)}{\mu(x)}\right)_{p},
\end{aligned}
$$

то есть

$$
\left(\frac{\eta(x)}{\mu(y)}\right)_{p}=\left(\frac{\eta(y)}{\mu(x)}\right)_{p}
$$

для всех $x, y \in \mathbf{G}_{\mathbf{0}}$.

Положим

$$
\mathscr{L}_{1}=d_{A} / \gamma * d_{B}
$$

Ясно, что $\mathscr{L}_{1}$ может быть вычислен противником по открытой информации за одно вычисление операции $*$ и одно деление в $Z\left[\zeta_{p}\right]$. То, что $d_{A}$ делится нацело на $\gamma$ (в смысле $\left.\mathbf{Z}\left[\zeta_{p}\right]\right)$ следует из определений $d_{A}$ и операции $*$.

Распишем $\mathscr{K}$ и $\mathscr{L}_{1}$ через обычное умножение, используя определение операции $*$, ее 
ассоциативность и свойства функций $\eta$ и $\mu$ :

$$
\begin{aligned}
\mathcal{K}= & a_{1} * d_{B} * a_{2} \\
= & a_{1} \cdot b_{1} \cdot \gamma \cdot b_{2} \cdot a_{2} \\
& \cdot\left(\frac{\eta\left(a_{1}\right)}{\mu\left(b_{1}\right)}\right)_{p} \cdot\left(\frac{\eta\left(a_{1}\right)}{\mu\left(b_{2}\right)}\right)_{p} \cdot\left(\frac{\eta\left(a_{1}\right)}{\mu(\gamma)}\right)_{p} \cdot\left(\frac{\eta\left(a_{1}\right)}{\mu\left(a_{2}\right)}\right)_{p} \cdot\left(\frac{\eta\left(b_{1}\right)}{\mu\left(b_{2}\right)}\right)_{p} \\
& \cdot\left(\frac{\eta\left(b_{1}\right)}{\mu(\gamma)}\right)_{p} \cdot\left(\frac{\eta\left(b_{1}\right)}{\mu\left(a_{2}\right)}\right)_{p} \cdot\left(\frac{\eta(\gamma)}{\mu\left(b_{2}\right)}\right)_{p} \cdot\left(\frac{\eta(\gamma)}{\mu\left(a_{2}\right)}\right)_{p} \cdot\left(\frac{\eta\left(b_{2}\right)}{\mu\left(a_{2}\right)}\right)_{p}, \\
\mathscr{L}_{1}= & \frac{d_{A}}{\gamma} * d_{B} \\
= & a_{1} \cdot b_{1} \cdot \gamma \cdot b_{2} \cdot a_{2} \\
& \cdot\left(\frac{\eta\left(a_{1}\right)}{\mu\left(b_{1}\right)}\right)_{p} \cdot\left(\frac{\eta\left(a_{1}\right)}{\mu(\gamma)}\right)_{p} \cdot\left(\frac{\eta\left(a_{1}\right)}{\mu\left(a_{2}\right)}\right)_{p} \cdot\left(\frac{\eta\left(a_{1}\right)}{\mu\left(b_{2}\right)}\right)_{p} \cdot\left(\frac{\eta\left(b_{1}\right)}{\mu\left(b_{2}\right)}\right)_{p} \\
& \cdot\left(\frac{\eta\left(b_{1}\right)}{\mu(\gamma)}\right)_{p} \cdot\left(\frac{\eta\left(a_{2}\right)}{\mu\left(b_{1}\right)}\right)_{p} \cdot\left(\frac{\eta(\gamma)}{\mu\left(a_{2}\right)}\right)_{p} \cdot\left(\frac{\eta(\gamma)}{\mu\left(b_{2}\right)}\right)_{p} \\
& \cdot\left(\frac{\eta\left(a_{2}\right)}{\mu\left(b_{2}\right)}\right)_{p} \cdot\left(\frac{\eta\left(a_{1} a_{2}\right)}{\mu(\gamma)}\right)_{p} \cdot
\end{aligned}
$$

Так как $a_{2}, b_{1}, b_{2} \in \mathbf{G}_{0}$, мы видим, что

$$
\begin{aligned}
& \left(\frac{\eta\left(a_{2}\right)}{\mu\left(b_{1}\right)}\right)_{p}=\left(\frac{\eta\left(b_{1}\right)}{\mu\left(a_{2}\right)}\right)_{p}, \\
& \left(\frac{\eta\left(a_{2}\right)}{\mu\left(b_{2}\right)}\right)_{p}=\left(\frac{\eta\left(b_{2}\right)}{\mu\left(a_{2}\right)}\right)_{p},
\end{aligned}
$$

поэтому

$$
\mathscr{L}_{1}=\mathscr{K} \cdot \tau_{1},
$$

где

$$
\tau_{1}=\left(\frac{\eta\left(a_{1} a_{2}\right)}{\mu(\gamma)}\right)_{p} .
$$

В силу определения операции $*$ и свойств функции $\eta$

$$
\begin{aligned}
\tau_{1} & =\left(\frac{\eta\left(a_{1} a_{2}\right)}{\mu(\gamma)}\right)_{p}=\left(\frac{\eta\left(d_{A} / \gamma\right)}{\mu(\gamma)}\right)_{p} \\
& =\frac{d_{A} / \gamma * \gamma}{d_{A} / \gamma \cdot \gamma}=\frac{d_{A} / \gamma * \gamma}{d_{A}} .
\end{aligned}
$$

Таким образом,

$$
\mathscr{H}=\frac{L_{1}}{\tau_{1}}=\frac{d_{A} / \gamma * d_{B}}{d_{A} / \gamma * \gamma} \cdot d_{A},
$$

то есть для вычисления $\mathscr{K}$ по открытой информации противнику нужно произвести два деления в $\mathbf{Z}\left[\zeta_{p}\right]$, одно умножение и два вычисления операции $*$, что и доказывает первое утверждение теоремы. 
Обратимся теперь ко второму утверждению теоремы. Выкладками, аналогичными вышеизложенным, можно показать, что

$$
\begin{aligned}
& \mathscr{K}=d_{A} \cdot d_{B} \cdot \tau_{0}, \\
& \mathscr{K}=\left(d_{A} * d_{B}\right) \cdot \tau_{1}{ }^{-1}, \\
& \mathscr{K}=\left(d_{B} * d_{A}\right) \cdot \tau_{2}{ }^{-1}
\end{aligned}
$$

Так как $d_{A}$ и $d_{B}$ находятся в открытом доступе, по этим формулам, зная $\mathscr{K}$, можно найти $\tau_{0}, \tau_{1}, \tau_{2}$.

\section{4. Криптоанализ схемы II для операции (2)}

Введем обозначение

$$
\mathbf{E}=\left\{x \in \mathbf{Z}\left[\zeta_{p}\right] \mid((x), \mathscr{g})=((x),(p))=1\right\}
$$

где $p$ - простое целое рациональное число. Тогда $*$ определена на $\mathbf{E} \times \mathbf{E}$. В разделе 2 мы показали, что данная операция $*$ является частным случаем операции, для которой формулировались теоремы в разделе 3, поэтому мы можем применять второе утверждение теоремы 1.

Определим функция $f(x), g(x)$ на $\mathbf{E}$, полагая

$$
\begin{array}{lll}
f(x)=i, & \text { где } i \in[0, p-1], & \left(\frac{N(x)}{\mathscr{J}}\right)_{p}=\zeta_{p}{ }^{i}, \\
g(x)=j, & \text { где } j \in[0, p-1], & \Phi_{p}(N(x)) \equiv j \quad(\bmod p) .
\end{array}
$$

Заметим, что при таком определении $f(x), g(x)$ справедливо равенство

$$
a * b=a \cdot b \cdot \zeta_{p}^{f(a) \cdot g(b)} .
$$

Предложение 1. Справедливы соотношения

$$
\begin{aligned}
f\left(\zeta_{p}\right) & =g\left(\zeta_{p}\right)=0 \\
f(x y) & \equiv f(x)+f(y) \quad(\bmod p) \\
g(x y) & \equiv g(x)+g(y) \quad(\bmod p)
\end{aligned}
$$

Доказательство. Докажем первое утверждение. Ясно, что

$$
N\left(\zeta_{p}\right)=1
$$

поэтому из определения частного Ферма следует, что

$$
\Phi_{p}\left(N\left(\zeta_{p}\right)\right)=\frac{1^{p-1}-1}{p} \quad(\bmod p)=0
$$

(то есть $g\left(\zeta_{p}\right)=0$ ) и из определения символа степенного вычета получаем, что

$$
f\left(\zeta_{p}\right)=0 .
$$


Второе утверждение верно в силу логарифмического свойства показателя и частных Ферма.

Рассмотрим следующие подмножества $\mathbf{E}$ :

$$
\begin{aligned}
H_{0,0} & =\{x \in \mathbf{E} \mid f(x)=g(x)=0\}, \\
H_{0} & =\{x \in \mathbf{E} \mid f(x)=0, g(x) \neq 0\}, \\
H_{p} & =\{x \in \mathbf{E} \mid f(x) \neq 0, g(x)=0\}, \\
H_{n} & =\left\{x \in \mathbf{E} \mid f(x) \cdot g(x)^{-1} \equiv n \quad(\bmod p)\right\}, \quad n=1, \ldots, p-1 .
\end{aligned}
$$

Заметим, что введенные множества попарно не пересекаются, а их объединение равно $\mathbf{E}$.

Предложение 2. Элементы множества $H_{0,0}$ коммутируют со всеми элементами Е. Элементы $x_{1}, x_{2} \notin H_{0,0}$ коммутируют тогда и только тогда, когда существует $n_{0} \in\{0,1, \ldots, p\}$ такое, что $x_{1}, x_{2} \in H_{n_{0}}$.

Доказательство. Из определения следует, что

$$
x_{1} * x_{2}=x_{2} * x_{1} \Longleftrightarrow f\left(x_{1}\right) \cdot g\left(x_{2}\right) \equiv f\left(x_{2}\right) \cdot g\left(x_{1}\right)
$$

(здесь и далее все сравнения по модулю $p$ ).

Если $x_{1} \in H_{0,0}$, то для всех $x_{2} \in \mathbf{E}$

$$
f\left(x_{1}\right) g\left(x_{2}\right) \equiv f\left(x_{2}\right) g\left(x_{1}\right) \equiv 0,
$$

поэтому

$$
x_{1} * x_{2}=x_{2} * x_{1} .
$$

Таким образом, первое утверждение доказано.

Пусть $x_{1}, x_{2} \in H_{n_{0}}$. Рассмотрим три случая.

Пусть $n_{0}=0$, тогда

$$
f\left(x_{1}\right)=f\left(x_{2}\right)=0 \text {, }
$$

поэтому

$$
f\left(x_{1}\right) \cdot g\left(x_{2}\right) \equiv f\left(x_{2}\right) \cdot g\left(x_{1}\right) \equiv 0 .
$$

Пусть $n_{0}=p$, тогда

$$
g\left(x_{1}\right)=g\left(x_{2}\right)=0 \text {. }
$$

поэтому

$$
f\left(x_{1}\right) \cdot g\left(x_{2}\right) \equiv f\left(x_{2}\right) \cdot g\left(x_{1}\right) \equiv 0 .
$$

Пусть $n_{0} \notin\{0, p\}$, тогда

$$
f\left(x_{1}\right) \cdot g\left(x_{1}\right)^{-1} \equiv f\left(x_{2}\right) \cdot g\left(x_{2}\right)^{-1} .
$$

Домножим обе части сравнения на $g\left(x_{1}\right) \cdot g\left(x_{2}\right) \not \equiv 0$, получим, что

$$
f\left(x_{1}\right) \cdot g\left(x_{2}\right) \equiv f\left(x_{2}\right) \cdot g\left(x_{1}\right) \text {. }
$$

Во всех трех случаях

$$
f\left(x_{1}\right) \cdot g\left(x_{2}\right) \equiv f\left(x_{2}\right) \cdot g\left(x_{1}\right),
$$


поэтому

$$
x_{1} * x_{2}=x_{2} * x_{1} .
$$

Таким образом, мы доказали, что если элементы лежат в одном множестве $H_{n_{0}}$, то они коммутируют.

Пусть

$$
x_{1} * x_{2}=x_{2} * x_{1}, \quad x_{1}, x_{2}, x_{3} \notin H_{0,0} .
$$

Тогда

$$
f\left(x_{1}\right) \cdot g\left(x_{2}\right) \equiv f\left(x_{2}\right) \cdot g\left(x_{1}\right) .
$$

Рассмотрим два возможных варианта.

Пусть $f\left(x_{1}\right) \cdot g\left(x_{2}\right) \equiv 0$, тогда в силу простоты $p$ либо $f\left(x_{1}\right) \equiv 0$, либо $g\left(x_{2}\right) \equiv 0$. Если $f\left(x_{1}\right) \equiv 0$, то (так как $\left.x_{1} \notin H_{0,0}\right)$ справедливо равенство $g\left(x_{1}\right) \not \equiv 0$. Кроме того,

$$
f\left(x_{2}\right) \cdot g\left(x_{1}\right) \equiv f\left(x_{1}\right) \cdot g\left(x_{2}\right) \equiv 0 \text {. }
$$

Поэтому $f\left(x_{2}\right) \equiv 0$, то есть $x_{2} \in H_{0}$ (так как $x_{2} \notin H_{0,0}$ ). Если же $g\left(x_{2}\right) \equiv 0$, то аналогично получим, что $x_{1}, x_{2} \in H_{p}$.

Пусть теперь

$$
f\left(x_{1}\right) \cdot g\left(x_{2}\right) \equiv f\left(x_{2}\right) \cdot g\left(x_{1}\right) \not \equiv 0 .
$$

Тогда обе части равенства можно разделить на ненулевое выражение $g\left(x_{1}\right) \cdot g\left(x_{2}\right)$. Получим, что

$$
f\left(x_{1}\right) \cdot g\left(x_{1}\right)^{-1} \equiv f\left(x_{2}\right) \cdot g\left(x_{2}\right)^{-1} \equiv n_{0} .
$$

то есть $x_{1}, x_{2} \in H_{n_{0}}$ для $n_{0} \in[1, p-1]$.

Из предложения 2 ясно, что при данном выборе операции $*$ подполугруппы $\mathbf{G}_{1}, \mathbf{G}_{2}$ будут подмножествами $H_{n_{1}} \sqcup H_{0,0}$ и $H_{n_{2}} \sqcup H_{0,0}$ для некоторых $n_{1} \neq n_{2}$ соответственно. Заметим, что $n_{i}$ нужно считать известными противнику, так как они могут быть вычислены по произвольному элементу из $\mathbf{G}_{i} \backslash H_{0,0}$.

Теорема 2. Для операчии (2) схема II является нестойкой.

Доказательство. Как мы уже отмечали, можно применить пункт 2 теоремы 1: если мы покажем, что по открытой информации можно быстро вычислять элемент

$$
\begin{aligned}
\tau_{1} & =\left(\frac{N\left(a_{2}\right)}{g}\right)_{p}^{\Phi_{p}\left(N\left(b_{1}\right)\right)} \cdot\left(\frac{N\left(b_{1}\right)}{g}\right)_{p}^{-\Phi_{p}\left(N\left(a_{2}\right)\right)} \\
& =\zeta_{p} f\left(a_{2}\right) \cdot g\left(b_{1}\right)-f\left(b_{1}\right) \cdot g\left(a_{2}\right)
\end{aligned}
$$

то этим мы докажем теорему. Таким образом достаточно показать, что по открытой информации можно быстро вычислить

$$
\rho=f\left(a_{2}\right) \cdot g\left(b_{1}\right)-f\left(b_{1}\right) \cdot g\left(a_{2}\right) \quad(\bmod p) .
$$

Будем считать, что $\mathbf{G}_{i}$ не содержат элементов из $H_{0,0}$ (случай, когда такие элементы есть, рассматривается аналогично). 
Введем обозначения

$$
\begin{aligned}
& x_{1}=f\left(a_{1}\right), \quad y_{1}=f\left(a_{2}\right), \quad z_{1}=f\left(b_{1}\right), \quad w_{1}=f\left(b_{2}\right), \quad c_{A}=f\left(d_{A}\right), \quad c_{B}=f\left(d_{B}\right), \\
& x_{2}=g\left(a_{1}\right), \quad y_{2}=g\left(a_{2}\right), \quad z_{2}=g\left(b_{1}\right), \quad w_{2}=g\left(b_{2}\right), \quad e_{A}=g\left(d_{A}\right), \quad e_{B}=g\left(d_{B}\right) \text {. }
\end{aligned}
$$

здесь $x_{i}, y_{i}, z_{i}, w_{i}$ - неизвестные, $c_{A}, e_{A}, c_{B}, e_{B}$ известны (они вычисляются по открытой информации исходя из определения). В таких обозначениях

$$
\rho=y_{1} z_{2}-z_{1} y_{2} \quad(\bmod p) .
$$

Посмотрим, какие уравнения с неизвестными $x_{i}, y_{i}, z_{i}, w_{i}$ мы можем составить. По предложению 1

$$
\begin{aligned}
& x_{1}+y_{1} \equiv c_{A}, \\
& x_{2}+y_{2} \equiv e_{A}, \\
& z_{1}+w_{1} \equiv c_{B}, \\
& z_{2}+w_{2} \equiv e_{B} .
\end{aligned}
$$

Далее нам нужно будет отдельно рассмотреть следующие случаи:

$$
\begin{array}{ll}
n_{i} \in\{0, p\}, & i=1,2, \\
n_{1} \in\{0, p\}, & n_{2} \notin\{0, p\} \text { или } n_{2} \in\{0, p\}, \quad n_{1} \notin\{0, p\}, \\
n_{i} \notin\{0, p\}, & i=1,2 .
\end{array}
$$

Мы подробно разберем только последний из названных случаев, в остальных все получается аналогично.

Напишем систему для $x_{i}, y_{i}, z_{i}, w_{i}$, получаемую непосредственно из определений множеств $H_{n_{1}}, H_{n_{2}}$. В данном случае она имеет вид

$$
\begin{aligned}
x_{1} & \equiv x_{2} \ldots n_{1}, \\
y_{1} & \equiv y_{2} \ldots n_{2}, \\
z_{1} & \equiv z_{2} \ldots n_{1}, \\
w_{1} & \equiv w_{2} \ldots n_{2} .
\end{aligned}
$$

Подставляя уравнения системы (4) в систему (3), получим, что

$$
\begin{aligned}
n_{1} x_{2}+n_{2} y_{2} & \equiv c_{A}, \\
x_{2}+y_{2} & \equiv e_{A}, \\
n_{2} w_{2} & \equiv c_{B}, \\
z_{2}+w_{2} & \equiv e_{B} .
\end{aligned}
$$

Это линейная система в поле $\mathbf{Z} / p \mathbf{Z}$ с определителем $\left(n_{1}-n_{2}\right)^{2} \not \equiv 0$. Поэтому она имеет единственное решение. Решая ее, получаем определенные выше неизвестные $x_{2}$, $y_{2}, z_{2}, w_{2}$ и далее по уравнениям системы (4) находим $x_{1}, y_{1}, z_{1}, w_{1}$.

В случаях, когда один или оба из $n_{1}, n_{2}$ лежат в $\{0, p\}$, мы получим другие системы вида (4) и соответственно другие системы вида (5). Но во всех случаях определитель системы вида (5) будет ненулевым и все неизвестные будут найдены. 


\section{Список литературы}

1. Сидельников В. М., Черепнев М. А., Ященко В. В., Системы открытого распределения ключей на основе некоммутативных полугрупп. ДАН СССР (1993), 332, 566-567.

2. Черепнев М. А., Схемы открытого распределения ключа на основе некоммутативной группы. Дискретная математика (2003) 15, №2, 47-51.

3. Черепнев М. А., Схемы открытого распределения ключа на основе некоммутативной операции. В сб.: Тез. докл. ХІІІ Международной конф. «Проблемы теоретической кибернетики». Казань, 2002, c. 190.

Статья поступила 9.09.2004. 